\section{(6) OPEN ACCESS}

\title{
Sex differences in cardiovascular outcome during progression of aortic valve stenosis
}

\author{
Dana Cramariuc, ${ }^{1}$ Barbara Patricia Rogge, ${ }^{1,2}$ Mai Tone Lønnebakken, ${ }^{2}$ Kurt Boman, $^{3}$ \\ Edda Bahlmann, ${ }^{4}$ Christa Gohlke-Bärwolf, ${ }^{5}$ John B Chambers, ${ }^{6}$ Terje R Pedersen, ${ }^{7}$ \\ Eva Gerdts ${ }^{2}$
}

\begin{abstract}
${ }^{1}$ Department of Heart Disease, Haukeland University Hospital, Bergen, Norway ${ }^{2}$ Department of Clinical Science, University of Bergen, Bergen, Norway

${ }^{3}$ Research unit, Medicine, Umeaa University, Skellefteaa, Sweden

${ }^{4}$ Asklepios Clinic St. Georg, Hamburg, Germany

${ }^{5}$ Herz-Zentrum Bad Krozingen, Bad Krozingen, Germany

${ }^{6}$ Guy's and St Thomas Hospitals, London, UK ${ }^{7}$ Department of Preventive Cardiology, Ullevål University Hopital, Oslo, Norway
\end{abstract}

\section{Correspondence to} Dr Dana Cramariuc, Department of Heart Disease, Haukeland University Hospital, Bergen NO-5021, Norway: dana.cramariuc@helse-bergen. no

Received 20 April 2014 Revised 17 September 2014 Accepted 21 September 2014 Published Online First 9 October 2014

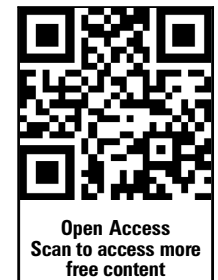

\section{CrossMark}

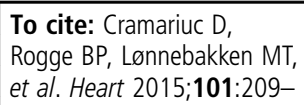

\section{ABSTRACT}

Objective Women with severe aortic valve stenosis

(AS) have better LV systolic function and more concentric LV geometry than their male counterparts. However, sex differences in cardiovascular (CV) outcome during progression of AS have not been reported from a longitudinal prospective study.

Methods Doppler echocardiography and CV events were recorded during a median of 4.0 years in 979 men and 632 women aged 28-86 (mean 67 \pm 10 ) years in the Simvastatin Ezetimibe in Aortic Stenosis (SEAS) study. LV systolic function was assessed by EF and midwall shortening (MWS). Study outcomes were ASrelated events, ischaemic CV events and total mortality. Results The annular cumulative incidence of AS events, ischaemic CV events and death was $8.1 \%, 3.4 \%$ and $2.8 \%$ in women, and $8.9 \%, 4.4 \%$ and $2.4 \%$ in men, respectively. Women and men had similar AS progression rate whether measured by peak jet velocity, mean gradient or valve area. In multivariate analyses, female sex independently predicted less reduction in LV MWS and EF during follow-up (both $\mathrm{p}<0.05$ ). In time-varying Cox analyses, women had a $40 \%$ lower rate of ischaemic CV events (95\% Cl $21 \%$ to $54 \%$ ), in particular, more than $50 \%$ lower rate of stroke and coronary artery bypass grafting, and a 31\% lower allcause mortality $(95 \% \mathrm{Cl} 1 \%$ to $51 \%)$, independent of active study treatment, age and hypertension, as well as time-varying valve area, low systolic function and abnormal LV geometry. AS event rate did not differ by sex.

Conclusions In the SEAS study, women and men had similar rates of AS progression and AS-related events. However, women had lower total mortality and ischaemic CV event rate than men independent of confounders.

Trial registration number ClinicalTrials.gov identifier: NCT00092677.

Aortic valve stenosis (AS) is present in $2 \%-7 \%$ of the population above 65 years of age and is the most common type of valvular heart disease requiring open heart surgery in developed countries. ${ }^{1} 2$ Management of AS is based upon assessment of clinical cardinal symptoms and of AS severity. ${ }^{3}$ Echocardiography is the key technique to assess AS severity and structural and functional consequences for the $\mathrm{LV}^{4}$

Previous cross-sectional studies in patients with severe, symptomatic AS have found that women present better LV systolic function than men, and more concentric LV geometry. ${ }^{5-8}$ Recently, women were found to have lower aortic valve calcium score by Agatston units on multislice CT than men for the same severity of AS. ${ }^{9}$ Furthermore, sex differences in comorbidities like hypertension, renal dysfunction and coronary artery disease are well known in patients with AS. ${ }^{10-12}$ However, although sex differences in comorbidities as well as in the pathophysiology of AS both at the aortic valve and LV level have been described, it is not clear whether women and men with AS have different progression rates or prognosis. ${ }^{13}$

The present, prospectively planned analysis aimed to compare AS progression rate and cardiovascular $(\mathrm{CV})$ outcome between women and men during progression of AS.

\section{METHODS}

\section{Study population}

The Simvastatin Ezetimibe in Aortic Stenosis (SEAS) study was a randomised, double-blind, placebo-controlled study which assessed the effect of combined treatment with simvastatin and ezetimibe on AS progression and CV morbidity and mortality in 1873 patients with initially asymptomatic, mild-moderate AS. ${ }^{14}$ Patients with known coronary artery disease, peripheral arterial disease, cerebrovascular disease, diabetes mellitus or any condition requiring lipid-lowering therapy were excluded from the SEAS study. The present prospectively planned analysis included the 1611 patients in whom LV geometry could be assessed on the echocardiogram both at baseline and at least one additional visit before occurrence of any major study outcome.

Hypertension was defined as history of hypertension, use of antihypertensive treatment reported by the attending physician or blood pressure $\geq 140$ / $90 \mathrm{~mm} \mathrm{Hg}$ at the baseline clinical visit. ${ }^{12}$ Blood tests, including measures of serum total and lowdensity lipoprotein (LDL)-cholesterol and assessment of kidney function by serum creatinine, were analysed at the SEAS core laboratory, PPD Global Central Labs (Zaventum, Belgium).

\section{Echocardiographic measurements}

Echocardiography was performed at baseline and then annually and before valve surgery at the 173 SEAS participating centres following a standardised performance protocol. ${ }^{10} 12$ The last study visit was 
defined as the last echocardiogram performed before a CV event in patients who experienced study endpoints or as the final study echocardiogram in those without events. All echocardiographic analyses were performed at the SEAS Echocardiography Core Laboratory at Haukeland University Hospital, Bergen, Norway, ${ }^{10} 12$ following current guidelines. ${ }^{411}$

\section{Assessment of AS severity}

Severity of AS was evaluated by peak aortic jet velocity, mean transvalvular gradient, aortic valve area and aortic valve area indexed for body surface area, as well as pressure recovery adjusted aortic valve area index, i.e. the energy loss index (ELI). ${ }^{4} 15$

\section{Assessment of LV geometry}

LV mass was calculated by the Devereux equation, ${ }^{11}$ and LV hypertrophy was considered present if $\mathrm{LV}$ mass/height ${ }^{2.7}$ $\geq 46.7 \mathrm{~g} / \mathrm{m}^{2.7}$ in women and $49.2 \mathrm{~g} / \mathrm{m}^{2.7}$ in men. ${ }^{16}$ Concentric geometry was considered present if relative wall thickness $\geq 0.43 .{ }^{11}$ Abnormal LV geometry was considered present if either LV hypertrophy or increased relative wall thickness was found.

\section{Assessment of LV systolic function}

LVEF was measured by the Simpson's biplane method. ${ }^{11}$ Gender-specific cut-off values for identification of low LV systolic function were used for EF and midwall shortening (MWS). ${ }^{17} \mathrm{EF}$ was considered low if $<51 \%$ in men and $<55 \%$ in women. ${ }^{17}$ MWS was calculated using validated equations and considered low if $<14 \%$ in men and $<16 \%$ in women. ${ }^{17}$

\section{Study outcomes}

The primary endpoint in the SEAS study was major CV events (combined AS-related events and ischaemic CV events). ${ }^{14}$ AS-related events included combined aortic valve replacement, hospitalisation for congestive heart failure due to AS or death from CV causes. Ischaemic CV events included combined nonfatal myocardial infarction, hospitalisation for unstable angina, coronary revascularisation at the time of aortic valve replacement, non-hemorrhagic stroke and CV death. The present analysis also assessed all-cause mortality, a tertiary study endpoint. All study endpoints were adjudicated by an independent classification committee.

\section{Statistical analyses}

Comparisons between women and men were done by $\chi^{2}$ test, unpaired Student's $t$ test, full-factorial two-way analysis of variance (ANOVA) with Sidak's posthoc test or ANOVA for repeated measures, as appropriate. Independent covariates of reduction in EF and MWS from baseline to the last study visit were identified in multivariate linear regression analyses, run with an enter procedure and collinearity diagnostics. The impact of sex on rate of AS-related and ischaemic CV events and on total mortality was tested in univariate and multivariate timevarying Cox proportional hazard analyses including age, hypertension and active study treatment, as well as AS severity by ELI, presence of low EF and low MWS, and abnormal LV geometry as time-varying covariates. Results are presented as HRs with 95\% CI, as well as plots of survival free of ischaemic CV events and overall survival in women and men with adjustment for the mean values of the other covariates included in the multivariate Cox models. Two-tailed $\mathrm{p} \leq 0.05$ was considered significant both in univariate and multivariate analyses.

\section{RESULTS}

\section{Progression of AS}

Women were older $(69 \pm 9$ vs. $66 \pm 10$ years), included more patients with hypertension (91\% vs. $83 \%)$ and had better renal function than men (all $\mathrm{p}<0.001$, table 1). Sixteen per cent of

Table 1 Clinical and echocardiographical characteristics of women and men at baseline and at the last study visit

\begin{tabular}{|c|c|c|c|c|}
\hline & \multicolumn{2}{|l|}{ Women (632) } & \multicolumn{2}{|l|}{ Men (979) } \\
\hline & Baseline & Last visit & Baseline & Last visit \\
\hline Body mass index $\left(\mathrm{kg} / \mathrm{m}^{2}\right)$ & $26.8 \pm 5.1 \ddagger$ & $26.4 \pm 5.5$ & $26.8 \pm 3.7 \S$ & $26.7 \pm 4.0$ \\
\hline Systolic blood pressure (mm Hg) & $146 \pm 20 \dagger \ddagger$ & $142 \pm 19^{*}$ & $144 \pm 20 \S$ & $138 \pm 18$ \\
\hline Diastolic blood pressure $(\mathrm{mm} \mathrm{Hg})$ & $82 \pm 10 \ddagger$ & $78 \pm 10$ & $82 \pm 10 \S$ & $79 \pm 10$ \\
\hline Heart rate (beats/min) & $68 \pm 11^{*}$ & $69 \pm 12^{*}$ & $64 \pm 11 \S$ & $66 \pm 13$ \\
\hline Total cholesterol (mmol/L) & $6.0 \pm 1.0^{*} \ddagger$ & $4.9 \pm 1.4^{*}$ & $5.5 \pm 0.9 \S$ & $4.4 \pm 1.3$ \\
\hline LDL cholesterol (mmol/L) & $3.7 \pm 0.9^{*} \ddagger$ & $2.6 \pm 1.3^{*}$ & $3.5 \pm 0.9 \S$ & $2.4 \pm 1.2$ \\
\hline Creatinine $(\mu \mathrm{mol} / \mathrm{L})$ & $84 \pm 13^{*} \ddagger$ & $77 \pm 17^{*}$ & $99 \pm 15 \S$ & $93 \pm 21$ \\
\hline Serum glucose (mmol/L) & $5.20 \pm 0.72^{*} \ddagger$ & $5.35 \pm 0.74^{*} \S$ & $5.34 \pm 0.84$ & $5.54 \pm 1.09$ \\
\hline Peak aortic jet velocity $(\mathrm{m} / \mathrm{s})$ & $3.07 \pm 0.53 \ddagger$ & $3.74 \pm 0.78$ & $3.10 \pm 0.55 \S$ & $3.67 \pm 0.76$ \\
\hline Mean transvalvular gradient $(\mathrm{mm} \mathrm{Hg})$ & $23 \pm 9 \ddagger$ & $35 \pm 15 t$ & $23 \pm 9 \S$ & $33 \pm 14$ \\
\hline Aortic valve area $\left(\mathrm{cm}^{2}\right)$ & $1.12 \pm 0.37^{\star} \ddagger$ & $0.98 \pm 0.36^{*}$ & $1.38 \pm 0.50 \S$ & $1.24 \pm 0.48$ \\
\hline Aortic valve area index $\left(\mathrm{cm}^{2} / \mathrm{m}^{2}\right)$ & $0.64 \pm 0.21^{*} \ddagger$ & $0.57 \pm 0.21$ * & $0.69 \pm 0.25 \S$ & $0.63 \pm 0.24$ \\
\hline ELI $\left(\mathrm{cm}^{2} / \mathrm{m}^{2}\right)$ & $0.86 \pm 0.40 \dagger \ddagger$ & $0.66 \pm 0.29^{*}$ & $0.92 \pm 0.47 \S$ & $0.73 \pm 0.33$ \\
\hline $\mathrm{EF}(\%)$ & $67 \pm 6 \ddagger$ & $65 \pm 6^{*}$ & $66 \pm 7 \S$ & $64 \pm 7$ \\
\hline MWS (\%) & $17.4 \pm 3.3^{*} \ddagger$ & $14.1 \pm 2.9 \dagger$ & $16.9 \pm 3.3 \S$ & $13.8 \pm 2.8$ \\
\hline LV mass index $\left(\mathrm{g} / \mathrm{m}^{2.7}\right)$ & $43 \pm 14^{*} \ddagger$ & $51 \pm 15^{*}$ & $47 \pm 15 \S$ & $55 \pm 17$ \\
\hline Relative wall thickness & $0.35 \pm 0.09 \ddagger$ & $0.48 \pm 0.12$ & $0.36 \pm 0.09 \S$ & $0.47 \pm 0.12$ \\
\hline Mitral regurgitation (mild/moderate) & $54 \% \dagger$ & $57 \% \dagger$ & $45 \% \S$ & $50 \%$ \\
\hline Aortic regurgitation (mild/moderate) & $56 \% \dagger \ddagger$ & $64 \% \dagger$ & $63 \% \S$ & $71 \%$ \\
\hline
\end{tabular}



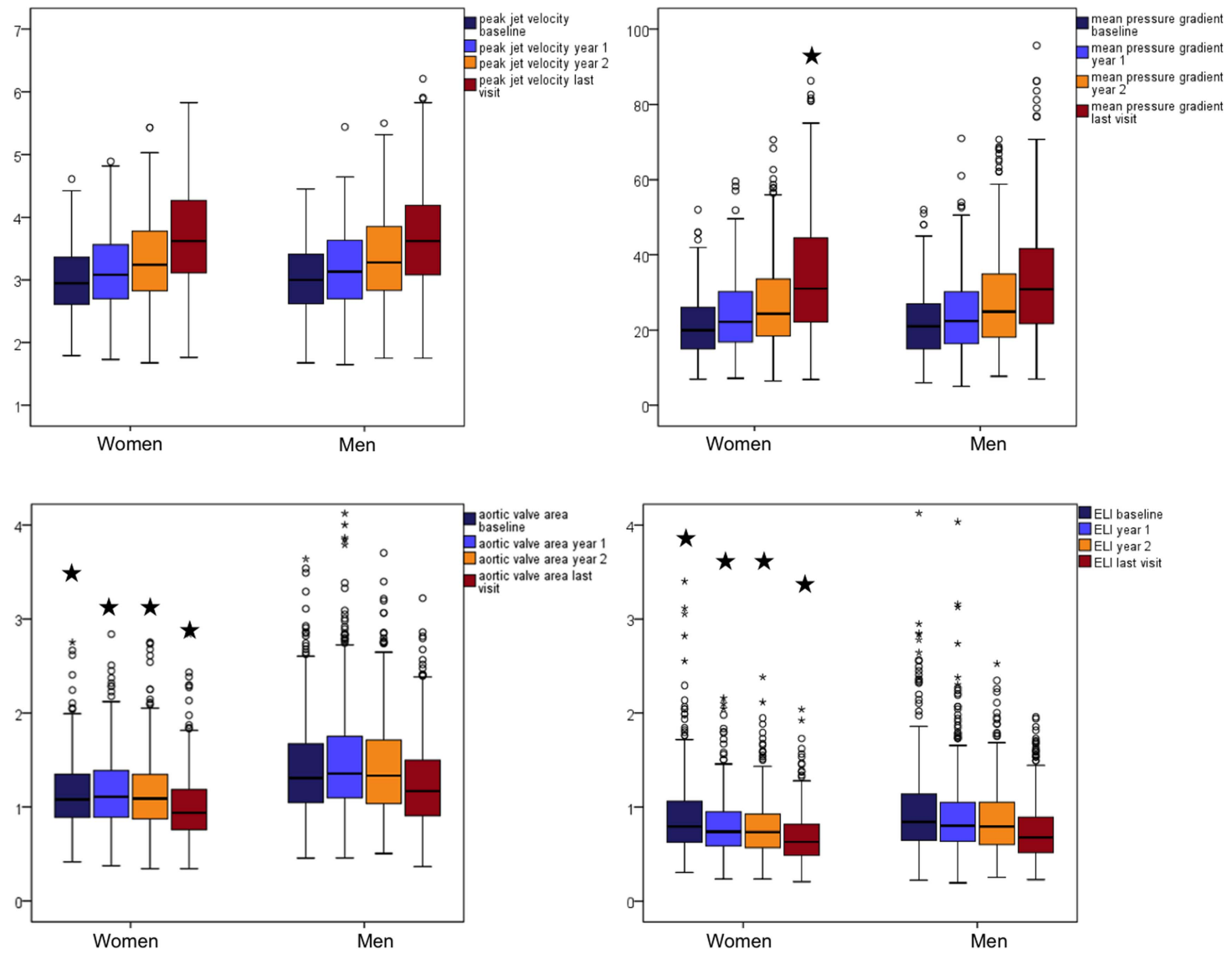

Figure 1 Progression of aortic valve stenosis in women and men assessed by 4 key variables: peak aortic jet velocity, mean pressure gradient, aortic valve area and energy loss index (ELI), at 4 different study visits: baseline, 1-year visit, 2-year visit and final visit. ${ }^{*} p<0.05$ for comparison between women and men at each visit.

women were smokers at study baseline vs. $21 \%$ of men $(\mathrm{p}<0.05)$. Women also had smaller aortic valve area both at inclusion and at the last study visit, also when adjusted for pressure recovery in the aortic root, indicating more severe AS (table 1 and figure 1).

Mild-moderate mitral regurgitation was more prevalent in women and mild-moderate aortic regurgitation more prevalent in men (both $\mathrm{p}<0.05$ ) (table 1). No patient had severe mitral or aortic valve regurgitation, reflecting the exclusion criteria in the SEAS study.

Although there was no stratification by sex in the randomisation procedure, randomisation to active study treatment did not differ between sexes: $50 \%$ of women on active treatment vs. $51 \%$ of men $(\mathrm{p}=0.61)$.

During a median of 4 years follow-up, AS progression rate did not differ between women and men, whether assessed from peak aortic jet velocity, mean transvalvular gradient, aortic valve area, aortic valve area index or ELI (table 2).

Low EF was uncommon in this population, reflecting the exclusion criteria used in the SEAS study. New-onset low EF was equally common in women and men during follow-up (figure 2). However, the absolute reduction in EF from baseline to the last examination was significantly larger in men $(2.6 \%$ vs. $1.4 \%$, $\mathrm{p}<0.01$ ) (table 1). Despite the low prevalence of reduced EF, low MWS was commonly found ( $p<0.001$ at all visits, figure 2 ).

In multivariate regression analyses, male sex independently predicted larger reduction both in EF and MWS during progression of AS (table 3). Baseline kidney function assessed by the serum creatinine level did not significantly influence reduction in EF or MWS in similar regression models.

\section{CV outcome}

During follow-up (median 4.0 years, IQR 3.0-4.2 years), a total of 554 major CV events occurred, among them 517 AS-related events and 260 ischaemic CV events. The ischaemic events included 56 strokes, 142 deaths from any cause (71 from CV causes), 28 non-fatal myocardial infarctions, 11 hospitalisations for unstable angina, 20 percutaneous coronary interventions and 142 coronary artery bypass revascularisations. Women had significantly lower risk of major CV events and ischaemic CV events (figure 3), also after adjustment for active study treatment, age and hypertension and time-varying ELI, low EF, low MWS and abnormal LV geometry in time-varying multivariate Cox analyses (table 4). Neither higher total cholesterol at baseline nor LDL cholesterol was associated with higher rate of

Table 2 Annual progression rate of aortic stenosis in women and men

\begin{tabular}{|c|c|c|c|}
\hline & $\begin{array}{l}\text { Women } \\
(632)\end{array}$ & $\begin{array}{l}\text { Men } \\
(979)\end{array}$ & $\begin{array}{l}p \\
\text { Value }\end{array}$ \\
\hline Peak aortic jet velocity (m/s/year) & $0.21 \pm 0.26$ & $0.19 \pm 0.31$ & 0.13 \\
\hline $\begin{array}{l}\text { Mean transvalvular gradient }(\mathrm{mm} \mathrm{Hg} / \\
\text { year) }\end{array}$ & $4 \pm 5$ & $4 \pm 6$ & 0.15 \\
\hline Aortic valve area $\left(\mathrm{cm}^{2} /\right.$ year $)$ & $0.04 \pm 0.14$ & $0.03 \pm 0.32$ & 0.77 \\
\hline Aortic valve area index $\left(\mathrm{cm}^{2} / \mathrm{m}^{2} /\right.$ year $)$ & $0.02 \pm 0.08$ & $0.02 \pm 0.16$ & 0.63 \\
\hline ELI $\left(\mathrm{cm}^{2} / \mathrm{m}^{2} /\right.$ year $)$ & $0.06 \pm 0.13$ & $0.05 \pm 0.23$ & 0.52 \\
\hline
\end{tabular}




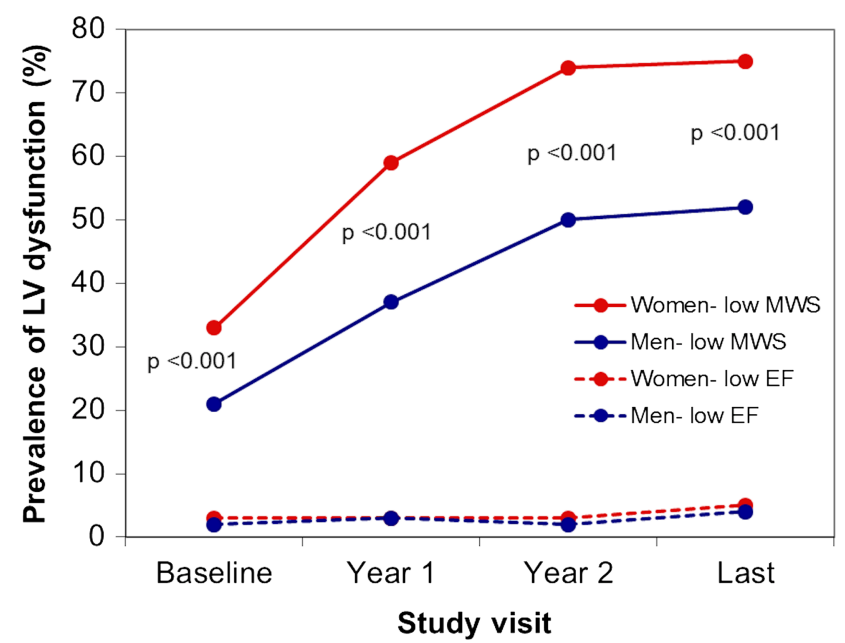

Figure 2 Prevalence of low midwall shortening (MWS) (continuous line) and low EF (stippled line) in women and men at different study visits. Mean values are adjusted for repeated measurements over time using full-factorial analysis of variance. $p$ Value of significance for comparison between women and men at each visit.

ischaemic $\mathrm{CV}$ events when added to the time-varying Cox model run in the whole study population as well as separately in women and men. When current smoking at baseline was added to these models, the results remained unchanged. Analysing the association between sex and specific types of ischaemic CV events using similar Cox models, female sex predicted more than 50\% lower rate of both stroke and coronary artery disease requiring concomitant bypass grafting at the time of aortic valve replacement (table 4). Women also had a 31\% lower all-cause mortality rate independent of covariates (table 4 and figure 4). The annular incidence of ischaemic CV events was $3.4 \%$ in women and $4.4 \%$ in men $(\mathrm{p}<0.007)$.

\section{DISCUSSION}

The present large, prospective, longitudinal study is the first to report on AS progression and associated CV events using a sexspecific approach. In particular, women and men had similar AS progression rates. However, women had lower total mortality despite more severe AS by echocardiographic indices and also

Table 3 Predictors of reduction in LVEF (multiple $R^{2}=0.39$, $p<0.001$ ) and MWS (multiple $R^{2}=0.51, p<0.001$ ) from baseline to the last study visit

\begin{tabular}{|c|c|c|c|c|}
\hline \multirow[b]{2}{*}{ Independent variables } & \multicolumn{2}{|c|}{ Reduction in EF } & \multicolumn{2}{|c|}{ Reduction in MWS } \\
\hline & B & p Value & B & p Value \\
\hline Male sex & 0.13 & $<0.001$ & 0.05 & 0.02 \\
\hline Age (years) & 0.02 & 0.42 & 0.09 & $<0.001$ \\
\hline Heart rate (bpm) & 0.08 & 0.001 & 0.03 & 0.14 \\
\hline Active study treatment & 0.03 & 0.25 & 0.03 & 0.12 \\
\hline Reduction in $\mathrm{ELI}\left(\mathrm{cm}^{2} / \mathrm{m}^{2}\right)$ & -0.03 & 0.24 & 0.003 & 0.87 \\
\hline Presence of aortic regurgitation & 0.01 & 0.68 & -0.01 & 0.81 \\
\hline Presence of mitral regurgitation & 0.05 & 0.02 & 0.01 & 0.76 \\
\hline LV hypertrophy & 0.12 & $<0.001$ & 0.06 & 0.006 \\
\hline Concentric LV geometry & -0.04 & 0.11 & 0.04 & 0.13 \\
\hline Baseline EF (\%) & 0.60 & $<0.001$ & -0.03 & 0.20 \\
\hline Baseline MWS (\%) & -0.03 & 0.37 & 0.76 & $<0.001$ \\
\hline
\end{tabular}

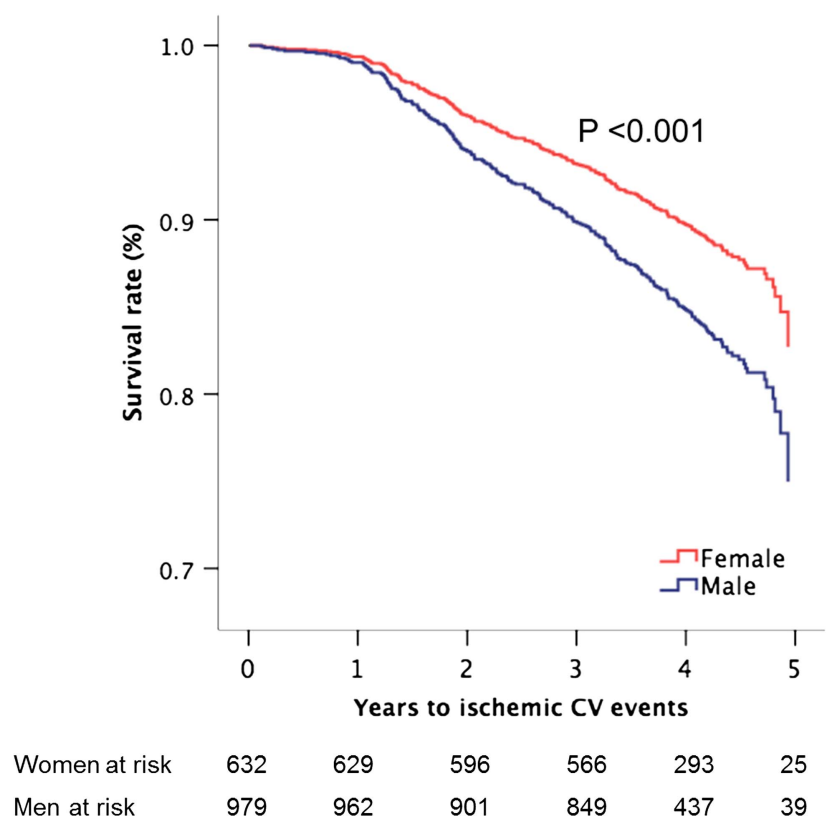

Figure 3 Survival free of ischaemic cardiovascular (CV) events in women and men during progression of aortic valve stenosis with adjustment for covariates (the means of age, hypertension, active study treatment, energy loss index, low EF and midwall shortening, and abnormal LV geometry) and $p$ value of significance based on Cox proportional hazard analyses.

lower rate of ischaemic CV events during a median of 4 years follow-up. The present study extends previous knowledge on sex differences from cross-sectional studies in AS which have demonstrated pathophysiological differences between women and men both at the aortic valve and LV level, including less valve calcification, more concentric LV geometry and extracellular myocardial fibrosis and higher indices of LV systolic function in women. ${ }^{5-7} 918-20$

\section{Progression of AS in women and men}

Few studies have compared AS progression in women and men. The Cardiovascular Health Study including echocardiographic follow-up over a mean of 5 years in 5621 subjects $\geq 65$ years of age reported that $9 \%$ of subjects with aortic valve sclerosis at the initial examination had progressed to AS on the follow-up

Table 4 The association of female sex with rates of major CV events, ischaemic CV events and death in time-varying multivariate Cox analyses adjusting for active study treatment, age and hypertension, as well as time-varying ELI, low EF, low MWS and abnormal LV geometry

\begin{tabular}{llc}
\hline & HR $(95 \% \mathrm{Cl})$ & $\mathrm{p}$ Value \\
\hline Major CV events & $0.83(0.69$ to 0.99$)$ & 0.04 \\
Aortic valve events & $0.85(0.70$ to 1.02$)$ & 0.08 \\
Ischaemic events & $0.60(0.46$ to 0.79$)$ & $<0.001$ \\
$\quad$ stroke & $0.47(0.26$ to 0.85$)$ & 0.01 \\
coronary artery bypass grafting & $0.49(0.34$ to 0.71$)$ & $<0.001$ \\
non-fatal myocardial infarction & $1.15(0.52$ to 2.54$)$ & 0.724 \\
hospitalisation for unstable angina & $0.62(0.17$ to 2.20$)$ & 0.456 \\
$\quad$ percutaneous coronary intervention & $0.62(0.21$ to 1.82$)$ & 0.386 \\
Death & $0.69(0.49$ to 0.99$)$ & 0.04 \\
\hline CV, cardiovascular; ELI, energy loss index; MWS, midwall shortening. &
\end{tabular}




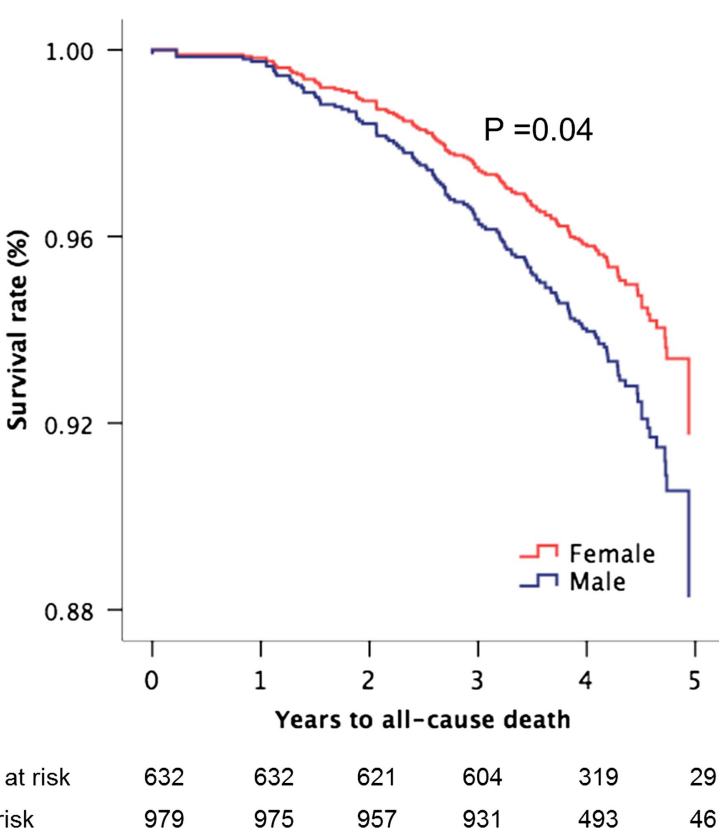

$\begin{array}{lllllll}\text { Women at risk } & 632 & 632 & 621 & 604 & 319 & 29 \\ \text { Men at risk } & 979 & 975 & 957 & 931 & 493 & 46\end{array}$

Figure 4 Overall survival in women and men during progression of aortic valve stenosis with adjustment for covariates (the means of age, hypertension, active study treatment, energy loss index, low EF and midwall shortening, and abnormal LV geometry) and $p$ value of significance based on Cox proportional hazard analysis.

examination, and progression to AS was threefold more common among men. ${ }^{21}$ By contrast, a retrospective study by Rosenhek et $a l^{22}$ including 73 women and 103 men with mildmoderate AS, found no interaction of sex with AS progression or outcome, while AS severity and aortic valve calcification by echocardiography were identified as independent predictors of progression to death or aortic valve replacement. Of note, using the more accurate Agatston score, aortic valve calcification was recently reported to be higher in men compared with women irrespective of AS severity. ${ }^{9}$ The present results add to these studies by demonstrating that the rate of progression of AS was comparable in women and men despite differences in age and prevalence of hypertension between sexes. However, compared with expected sex differences in incidence of CV events based upon general Norwegian population, the observed difference in incidence of ischaemic CV events was much less in the present study population. ${ }^{23}$ This finding probably reflects that AS often is part of a systemic atherosclerotic disease. ${ }^{24}$

\section{Sex-differences in CV outcomes in AS}

Sex-related differences in atherosclerosis progression, plaque composition and prevalence of microvascular disease have been well documented. ${ }^{25}$ Obstructive coronary artery disease and stroke are both more prevalent among men in the general population $<75$ years of age. ${ }^{25}$ Recently, superior LV hypertrophy regression and outcome were reported in elderly women compared with men undergoing transcutaneous aortic valve replacement from the prospective Placement of Aortic Transcatheter Valve (PARTNER) trial. ${ }^{26}$ The present results extend current knowledge by demonstrating that also after adjusting for known confounders, women had more than 50\% lower rate of ischaemic CV events, like stroke and coronary artery bypass surgery, as well as $31 \%$ lower total mortality during a median of 4 years follow-up. Of note, this was observed despite more severe AS from echocardiographic indices and higher prevalence of hypertension among women, both well-known factors associated with impaired prognosis in $\mathrm{AS}^{27} 28$ Reduced LVEF to $<50 \%$ is a guideline indication for valve replacement in severe AS. ${ }^{27}$ As demonstrated, men experienced more reduction in EF and more subclinical coronary artery disease during follow-up, but low EF was very uncommon, probably reflecting the SEAS study exclusion criteria. These preoperative differences may help explain the observed higher LVEF and enhanced hypertrophy regression in women in previous studies after aortic valve replacement for $\mathrm{AS},{ }^{18} 19^{26}$ as well as the recent findings of better survival in elderly women undergoing transcatheter aortic valve replacement in a retrospective sub-analysis from the PARTNER trial. ${ }^{26}$

\section{Study limitations}

This report is based on the prospective follow-up of a selected population of patients with AS and without diabetes mellitus, clinical manifest vascular disease, dyslipidaemia, or other significant valvular disease participating in the SEAS study. Exclusion of patients with diabetes mellitus was done based upon known history of diabetes. Inclusion of some patients with subclinical diabetes mellitus might have occurred, since HbA1c was not measured. Extrapolation of the results to other types of patients with AS should be done with caution. However, in patients with AS with clustering of CV risk factors or established CV disease, even higher incidence of ischaemic CV events is to be expected, and active CV prevention may be even more effective.

In conclusion, the present results demonstrate that women and men have similar rates of AS progression and AS-related events. However, women have lower total mortality and

\section{Key messages}

What is known on this subject?

Aortic stenosis (AS) progression rate and cardiovascular (CV) outcome during AS progression have not been reported individually for women and men from a prospective, longitudinal study.

\section{What might this study add?}

This study documents that AS progression rate is similar in women and men. Women had lower rate of ischaemic CV events and total death than men during a median of 4 years follow-up independent of confounding factors like age, hypertension, AS severity, LV geometry, systolic function and lipid lowering treatment. However, the observed sex difference in rate of ischaemic CV events was much smaller than expected from population-based studies, with an absolute annular incidence of $3.4 \%$ in women and $4.4 \%$ in men, respectively, meaning only $30 \%$ lower absolute incidence in women compared with men. It is well known that AS is associated with systemic atherosclerosis. Of note, the most common type of ischaemic CV event in our study was concomitant coronary artery bypass grafting during planned surgical aortic valve replacement. The total number of acute myocardial infarctions was low in this population during the study period with an absolute annular incidence of $0.5 \%$ vs. $0.4 \%$ in women and men, respectively (not significant).

\section{How might this impact on clinical practice?} Our findings underscore that progression of AS occurs at comparable rates in women and men, and guideline-recommended follow-up should be provided in both sexes. 
ischaemic CV event rates compared with men during progression of AS, even after adjustment for confounding factors like differences in age, prevalence of hypertension, AS severity, LV geometry and LV systolic function.

Contributors DC, BPR, MTL and EG were involved in the conception and design, as well as analysis and interpretation of data. All coauthors have revised the manuscript critically and approved it for submission to Heart.

Funding The SEAS (Simvastatin Ezetimibe in Aortic Stenosis) Echocardiography Core Laboratory was supported by MSP Singapore Company, LLC, Singapore, a partnership between Merck Co, and the Schering-Plough Corporation.

Competing interests $E G$ received steering committee honorarium and funding for the echocardiography core laboratory from Merck Schering Plough. TRP has received consulting fees and speaker honoraria from Merck Sharp and Dohme. KB received steering committee honorarium and speaker honoraria from Merck Schering Plough. The other authors have no disclosures related to the present study.

Patient consent Obtained.

Ethics approval The study was approved by all regional ethics committees.

Provenance and peer review Not commissioned; externally peer reviewed.

Open Access This is an Open Access article distributed in accordance with the Creative Commons Attribution Non Commercial (CC BY-NC 4.0) license, which permits others to distribute, remix, adapt, build upon this work non-commercially, and license their derivative works on different terms, provided the original work is properly cited and the use is non-commercial. See: http://creativecommons.org/ licenses/by-nc/4.0/

\section{REFERENCES}

1 lung $B$, Baron $G$, Butchart $E G$, et al. A prospective survey of patients with valvular heart disease in Europe: The Euro Heart Survey on Valvular Heart Disease. Eur Heart J 2003;24:1231-43.

2 Nkomo VT, Gardin JM, Skelton TN, et al. Burden of valvular heart diseases: a population-based study. Lancet 2006;368:1005-11.

3 Otto CM. Valvular aortic stenosis: disease severity and timing of intervention. J Am Coll Cardiol 2006;47:2141-51.

4 Baumgartner $\mathrm{H}$, Hung J, Bermejo J, et al. Echocardiographic assessment of valve stenosis: EAE/ASE recommendations for clinical practice. J Am Soc Echocardiogr 2009;22:1-23.

5 Aurigemma GP, Silver KH, McLaughlin $\mathrm{M}$, et al. Impact of chamber geometry and gender on left ventricular systolic function in patients $>60$ years of age with aortic stenosis. Am J Cardiol 1994;74:794-8.

6 Carroll JD, Carroll EP, Feldman T, et al. Sex-associated differences in left ventricular function in aortic stenosis of the elderly. Circulation 1992;86:1099-107.

7 Douglas PS, Otto CM, Mickel MC, et al. Gender differences in left ventricle geometry and function in patients undergoing balloon dilatation of the aortic valve for isolated aortic stenosis. NHLBI Balloon Valvuloplasty Registry. Br Heart J 1995;73:548-54

8 Cramariuc D, Gerdts E, Davidsen ES, et al. Myocardial deformation in aortic valve stenosis: relation to left ventricular geometry. Heart 2010;96:106-12.

9 Aggarwal SR, Clavel MA, Messika-Zeitoun D, et al. Sex differences in aortic valve calcification measured by multidetector computed tomography in aortic stenosis. Circ Cardiovasc Imaging 2013;6:40-7.
10 Cramariuc D, Rieck AE, Staal EM, et al. Factors influencing left ventricular structure and stress-corrected systolic function in men and women with asymptomatic aortic valve stenosis (a SEAS Substudy). Am J Cardiol 2008;101:510-15.

11 Lang RM, Bierig M, Devereux RB, et al. Recommendations for chamber quantification. Eur J Echocardiogr 2006;7:79-108.

12 Rieck $A E$, Cramariuc D, Staal EM, et al. Impact of hypertension on left ventricular structure in patients with asymptomatic aortic valve stenosis (a SEAS substudy). J Hypertens 2010;28:377-83.

13 Shames S, Gillam LD. Sex differences in aortic valve calcification. Circ Cardiovasc Imaging 2013;6:8-10.

14 Rossebo $A B$, Pedersen TR, Boman $K$, et al. Intensive lipid lowering with simvastatin and ezetimibe in aortic stenosis. N Engl J Med 2008;359:1343-56.

15 Bahlmann E, Gerdts E, Cramariuc D, et al. Prognostic value of energy loss index in asymptomatic aortic stenosis. Circulation 2013;127:1149-56.

16 de Simone G, Izzo R, Chinali M, et al. Does information on systolic and diastolic function improve prediction of a cardiovascular event by left ventricular hypertrophy in arterial hypertension? Hypertension 2010;56:99-104.

17 Bella JN, Palmieri V, Roman MJ, et al. Gender differences in left ventricular systolic function in American Indians (from the Strong Heart Study). Am J Cardiol 2006;98:834-7

18 Gerdts E, Stangeland L, Engedal H, et al. Postoperative Doppler echocardiographic evaluation in different sizes of Medtronic-Hall, Biocor and Carpentier-Edwards S.A.V. prosthetic aortic valves. Scand J Thorac Cardiovasc Surg 1994;28:25-9.

19 Petrov G, Regitz-Zagrosek V, Lehmkuhl E, et al. Regression of myocardial hypertrophy after aortic valve replacement: faster in women? Circulation 2010;122 (11 Suppl):S23-8.

20 Villari B, Campbell SE, Schneider J, et al. Sex-dependent differences in left ventricular function and structure in chronic pressure overload. Eur Heart $J$ 1995;16:1410-19.

21 Novaro GM, Katz R, Aviles RJ, et al. Clinical factors, but not C-reactive protein, predict progression of calcific aortic-valve disease: the Cardiovascular Health Study. J Am Coll Cardiol 2007;50:1992-8.

22 Rosenhek R, Klaar U, Schemper M, et al. Mild and moderate aortic stenosis. Natural history and risk stratification by echocardiography. Eur Heart J 2004;25:199-205.

23 Mannsverk J, Wilsgaard T, Njolstad I, et al. Age and gender differences in incidence and case fatality trends for myocardial infarction: a 30-year follow-up. The Tromso Study. Eur J Prev Cardiol 2012;19:927-34.

24 Pibarot P, Dumesnil JG. Assessment of aortic stenosis severity: check the valve but don't forget the arteries! Heart 2007;93:780-2.

25 Roger VL, Go AS, Lloyd-Jones DM, et al. Heart disease and stroke statistics-2012 update: a report from the American Heart Association. Circulation 2012;125: e2-220.

26 Williams M, Kodali SK, Hahn RT, et al. Sex-related differences in outcomes following transcatheter or surgical aortic valve replacement in patients with severe aortic stenosis: Insights From The PARTNER Trial (Placement of Aortic Transcatheter Valve). J Am Coll Cardiol 2014;63:1522-8.

27 Nishimura RA, Otto CM, Bonow RO, et al. 2014 AHA/ACC guideline for the management of patients with valvular heart disease: a report of the American College of Cardiology/American Heart Association Task Force on Practice Guidelines. J Am Coll Cardiol 2014;63:2438-88.

28 Rieck AE, Cramariuc D, Boman K, et al. Hypertension in aortic stenosis: implications for left ventricular structure and cardiovascular events. Hypertension 2012;60:90-7. 